\title{
THEORY OF DISORDERED ALLOYS IN THE TIGHT BINDING APPROXIMATION. I
}

\author{
EDWARD A. STERN* \\ Royal Society Mond Laboratory, University of Cambridge $\boldsymbol{\dagger}$ \\ (Received 30 October 1964; \\ in revised form 25 February 1965)
}

\begin{abstract}
This paper gives a theoretical treatment of the wave functions of electrons in disordered binary alloys in the tight binding approximation (TBA). For simplicity only s-type atomic wave functions are assumed though the results can be qualitatively applied to the d-bands of transition metals. The most important result is that the constituents in the alloy have in general a different number of TBA electrons than in the pure metals. This charging effect depends on the composition of the alloy and the parameter $\delta_{0}=\varepsilon_{21}{ }^{\prime} / \Delta$, where $\varepsilon_{21}{ }^{\prime}$ is the magnitude of the difference of the atomic energies of the two constituents in the alloy and $\Delta$ is the half-width of the band. The charging effect, in turn, changes the atomic energies of the constituents in the alloy from their values in the pure metals requiring a self-consistent solution. We solve in the TBA the simplest possible case which shows a charging effect, that of an ordered alloy with equal composition of the two constituents. The charging effect and $\delta_{0}$ are numerically found selfconsistently for the two cases of equal valency and non-equal valency constituents in the ordered alloy. In this alloy a band gap always appears for any finite value of $\delta_{0}$. This is not true for a disordered alloy; for this we use a wave function for which scattering can be neglected in the two extreme cases when $\delta_{0}$ is small or large compared to unity. In the former case there is no band gap while in the latter the band splits into two, each sub-band depending on the properties of only one type of atom. In the former case, with $N_{1}$ type one atoms and $N_{2}$ type two atoms, the amplitude of the wave function is about equal on the two types of atoms throughout the band, differing only of order $\delta_{0}$, and the rigid band model applies. In the latter case the amplitude is always mainly on either one or the other type of atom. These conclusions follow if the values of the band widths of the pure metals are approximately equal. When they differ, the character of the band near the top is determined only by the properties of the atom for which the band is wide in the pure state. This has application to alloys of Pd with $\mathrm{Ag}$ and Pt with $\mathrm{Au}$ where it is shown that for small concentrations of $\mathrm{Ag}$ and Au the specific heat
\end{abstract}

* Guggenheim Fellow, 1963-1964.

† Temporary address during Sabbatical leave.

Permanent address: Department of Physics and Astronomy, University of Maryland. 
of the alloy is a correct measure of the density of states of the d-band of the pure transition metal. For large concentrations, scattering becomes important, changing the shape of the $d$-band in the alloy, and explaining the tail found in the experimental results. Orthogonalization of the alloy wave functions insures that for a completely filled band all atoms have the same average electronic charge. However, when the band is not completely full there is the above mentioned net electronic charge difference between the two types of atoms which for small $\delta_{0}$ is proportional to $\delta_{0}$. This charge difference is measured by $\Delta \rho_{i}$, the difference between the average charge and the charge on the $i-t h$ type atom (cf. equation (A10)), and is the only way to produce a difference in electronic charge on the two constituents as may be required by different numbers of $d$-band electrons per atom. The constant of proportionality between $\Delta \rho_{i}$ and $\delta_{0}$ is largest when the Fermi level of the alloy is near the middle of the band tending to zero as the band empties or fills. Thus alloys whose Fermi level is near the middle of the band are more likely to have small $\delta_{0}$ and satisfy the rigid band model than those whose Fermi levels are near the top or bottom of the band. It is this $\Delta \rho_{i}$ which must be solved self-consistently with $\delta_{0}$ and in a general solution one expects deviations from charge neutrality on a given atom type ( $\sigma$ in equation 13') which changes the atomic energy of the TBA electrons. The variation of the atomic energy with $\sigma$ must include, in any real transition metal, effects of screening by the s-electrons, which, as recent investigations show, has a major effect on the energies concerned. The theory neglects all spin dependent interactions. Although only the TBA is treated here, general physical reasoning indicates that the charging effect is present in all types of alloys, and must be included in a self-consistent manner when determining the lattice potential seen by an electron.

\section{Introduction}

Our present knowledge of the electronic structure of normal metals has progressed spectacularly in the last few years and, by means of various powerful techniques, the shape of the Fermi surface for many normal metals has been very accurately determined. The electronic structure of the transition and rare earth metals is more complicated and the detailed information on the shape of the Fermi surface has yet to be obtained [1]. Another important parameter is the shape of the density of states per unit energy interval. One technique that has been used to determine the density of states, especially in the transition metals, is to measure a property (magnetic susceptibility, specific heat) of an alloy made by combining another metal with the one under study [2]. The alloying metal is chosen to have a different valency than the metal under study so as to change the ratio of electrons to atoms $(e / a)$. The experimental results are then, when possible, interpreted in the framework of the rigid band model which assumes that alloying does not change the shape of the density of states and only changes the $e / a$. At present, it is not clear theoretically when the rigid band model of transition metals is a valid approximation and when it fails. To settle this question requires an investigation into the band structure of alloys.

The purpose of this paper is thus to understand the structure of the $d$-band in the transition metal alloys. There is, of course, always some hybridization of the atomic $d$-states with the atomic $s$-states in the transition metals, but, as various authors have stressed, it is still a useful approximation for many purposes to neglect this [1], and we give no discussion of hybridization in the paper. For our purpose it is sufficient to treat the $d$-band in the tight binding approximation (TBA), and to solve the simpler problem of an alloy in the TBA with an $s$ band instead of a $d$-band. 
The next section (Section 2) presents some general physical considerations as an introduction to the theory of alloys in the tight-binding approximation. Section 3 follows by solving the problem of a 50\%-50\% ordered binary alloy exactly in the TEA. This problem illustrates many of the physical concepts necessary in understanding transition metal alloys. In Section 4 the properties of disordered binary alloys are calculated in the Bloch limit (small $\delta_{0}$ ) as defined in that section. The following section (Section 5) calculates the properties of a binary disordered alloy in the other extreme limit, the atomic limit (large $\delta_{0}$ ). In Section 6 the application of these physical ideas to real transition metal alloys is discussed. Section 7 is a summary of the paper and a comparison with the related work of other authors.

\section{General Considerations}

In the usual TBA the wave functions in the vicinity of an ion in a solid are assumed to be closely approximated by the atomic wave function normalized to unity. When we consider an alloy system this picture has to be modified. Consider a two component alloy of approximately equal composition. Imagine the alloy is built up by first placing the bare ions in their final places in the lattice without the electrons which are to be treated by the TBA. Then the TBA electrons are added to the solid. At first the electrons, to minimize their energy, will concentrate on the ion with the lowest atomic energy level. We call this ion type 1 . However, as more and more electrons are added, the relatively greater charge on the type 1 ions will increase till the self-consistent field due to the TBA electrons and the ion core raises the atomic energy level on atoms 1 near to that of the other ions, called type 2. Then further addition of electrons will add almost equal charge to the two types of atoms. At the end of this process the solid would consist of type 1 atoms with a net negative charge and type 2 atoms with a net positive charge. Finally, when the valence electrons are added they will screen the charges, but since the screening cannot be complete the TBA electrons will still see some of the effects of charging. The appropriate potential then seen by the TBA electrons is not the atomic potential but a modified one due to the build up of charge which tends to equalize the energies of the states of type 1 and type 2 atoms. It should be emphasized that the alloy problem in the TBA, in contrast to the pure metal case, must explicitly include this many-body effect of charging. This charging process is inhibited if the available energy levels of type 1 atoms become filled before all electrons are inserted and then all additional electrons are forced by the Pauli exclusion principle to populate type 2 atoms. The $d$-electrons in a gold-silver alloy are an example of this.

Although the discussion here specifically mentions the TBA, the same reasoning should hold and a charging effect is expected for any alloy.

The method used throughout most of this paper is to make an educated guess as to the appropriate type of wave function for the alloy, and then to use the minimization of the energy to determine all unknown parameters. The method will work best when the wave functions used have only a small number of unknowns. Afterwards various checks are made to see how good the wave function actually is.

As discussed, the many body effect of charging of the ion cores in alloys must be treated explicitly. We do so in this paper only in the simplest approximation, the Hartree-Fock approximation. This approximation is consistent with the other approximation made in this paper, the one-electron approximation. The usefulness of the one-electron approximation for the $a$-bands of the transition metals has been justified by Mott [1].

In the one-electron approximation, the wave function of the solid is a slater determinant of 
one electron wave functions $\psi(\mathbf{r})$. The functions $\psi(\mathbf{r})$ are solutions of a one-electron Schrodinger equation

$$
\left(\mathbf{P}^{2} / 2 m+V(\mathbf{r})\right) \psi(\mathbf{r})=\varepsilon \psi(\mathbf{r})
$$

where $\mathbf{P}=(\hbar / i) \nabla$ and $V(\mathbf{r})$ is the effective potential seen by the electron including effects of exchange and charging. To simplify the problem we will in this paper neglect all spin dependent contributions to $V(\mathbf{r})$. Thus the function $\psi(\mathbf{r})$ is a product of a spin dependent and a spatial dependent part. In what follows, the spin dependent part of $\psi(\mathbf{r})$ will not be explicitly stated except when necessary.

\section{Ordered Binary Alloy}

The simplest case of a 50\%-50\% binary ordered alloy consisting of a type 1 and a type 2 atom is considered. Both the cases where the two types of atoms have the same and different numbers of TBA electrons per atom will be considered. The ordered alloy is a periodic array of atoms with a unit cell consisting of one each of a type 1 and type 2 atom. The TBA wave function $\psi(\mathbf{r})$ is a Bloch wave function of the form

$$
\psi_{\mathbf{k}}(\mathbf{r})=\sum_{\mathbf{a}} e^{i \mathbf{k} \cdot \mathbf{R}_{\mathbf{a}}}\left[\phi_{1}\left(\mathbf{r}-\mathbf{R}_{\mathbf{a}}\right)+b e^{i \mathbf{k} \cdot \mathbf{q}} \phi_{2}\left(\mathbf{r}-\mathbf{R}_{\mathbf{a}}-\mathbf{q}\right)\right]
$$

Here $\mathbf{R}_{a}$ is the position of the type one atoms and $\mathbf{q}$ is the relative position of the type 2 atom referred to the type 1 atom in the unit cell. $\phi_{1}$ and $\phi_{2}$ are the atomic wave functions for the type 1 and 2 atoms, respectively. Because of the charging effects the atomic wave-functions in the solid will be different from those in the isolated atom. The $\phi_{1}$ and $\phi_{2}$ are the atomic wave functions in the solid which are solution of the Hamiltonians

$$
\begin{aligned}
& {\left[\left(\mathbf{P}^{2} / 2 m\right)+U_{1}\left(\mathbf{r}-\mathbf{R}_{\mathbf{a}}\right)\right] \phi_{1}\left(\mathbf{r}-\mathbf{R}_{\mathbf{a}}\right)=\varepsilon_{1} \Phi_{1}\left(\mathbf{r}-\mathbf{R}_{\mathbf{a}}\right)} \\
& {\left[\left(\mathbf{P}^{2} / 2 m\right)+U_{2}\left(\mathbf{r}-\mathbf{R}_{\mathbf{a}}-\mathbf{q}\right)\right] \Phi_{2}\left(\mathbf{r}-\mathbf{R}_{\mathbf{a}}-\mathbf{q}\right)=\varepsilon_{2} \Phi_{2}\left(\mathbf{r}-\mathbf{R}_{\mathbf{a}}-\mathbf{q}\right)}
\end{aligned}
$$

The $U_{1}$ and $U_{2}$ are not the isolated atomic potentials but the ones in the solid including the charging effects. The constant $b$ is to be determined by minimizing the energy.

We generalize the usual equations of the TBA to our case and assume that

$$
\begin{aligned}
& \int \varphi_{1}^{*}\left(\mathbf{r}-\mathbf{R}_{\mathbf{a}}\right) \varphi_{1}\left(\mathbf{r}-\mathbf{R}_{\mathbf{b}}\right) d^{3} r=\delta_{a b} \\
& \int \varphi_{1}^{*}\left(\mathbf{r}-\mathbf{R}_{\mathbf{a}}\right) \varphi_{2}\left(\mathbf{r}-\mathbf{R}_{\mathbf{s}}\right) d^{3} r=0 \\
& \int \Phi_{2}^{*}\left(\mathbf{r}-\mathbf{R}_{\mathbf{s}}\right) \varphi_{2}\left(\mathbf{r}-\mathbf{R}_{\mathbf{t}}\right) d^{3} r=\delta_{s t}
\end{aligned}
$$

the position of the type two atoms is abbreviated by $\mathbf{R}_{\mathbf{s}}$ and $\mathbf{R}_{\mathbf{t}}$. The energy corresponding to $\psi_{k}$
is 


$$
\begin{aligned}
\left(1+b b^{*}\right) \varepsilon_{k}= & \varepsilon_{1}^{\prime}+\varepsilon_{2}{ }^{\prime} b b^{*}+\sum_{c} \alpha_{1} e^{i \mathbf{k} \cdot\left(\mathbf{R}_{\mathbf{a}}-\mathbf{R}_{\mathbf{c}}\right)}+b^{*} \sum_{s} \alpha_{12} e^{i \mathbf{k} \cdot\left(\mathbf{R}_{\mathbf{a}}-\mathbf{R}_{\mathbf{s}}\right)} \\
& +b b^{*} \sum_{t}{\underset{\alpha}{2}}^{i \mathbf{k} \cdot\left(\mathbf{R}_{\mathbf{s}}-\mathbf{R}_{\mathbf{t}}\right)}+\underset{\mathbf{a}}{\sum_{\alpha_{2}} e^{i \mathbf{k} \cdot\left(\mathbf{R}_{\mathbf{s}}-\mathbf{R}_{\mathbf{a}}\right)}}
\end{aligned}
$$

The convention is used that subscripts of letters near the beginning of the alphabet denote type 1 atoms and subscripts of letters near the end of the alphabet denote type 2 atoms.

The sums in (5) are over the appropriate neighbors of the two atoms in a unit cell. In addition

$$
\begin{aligned}
& \varepsilon_{1}^{\prime}=\varepsilon_{1}+\int \Phi_{1}^{*}\left(\mathbf{r}-\mathbf{R}_{\mathbf{a}}\right) V_{1 a} \Phi_{1}\left(\mathbf{r}-\mathbf{R}_{\mathbf{a}}\right) d^{3} r \\
& \varepsilon_{2}^{\prime}=\varepsilon_{2}+\int \Phi_{2}^{*}\left(\mathbf{r}-\mathbf{R}_{\mathrm{s}}\right) V_{2 s} \Phi_{2}\left(\mathbf{r}-\mathbf{R}_{\mathrm{s}}\right) d^{3} r \\
& \alpha_{1}=\int \Phi_{1}^{*}\left(\mathbf{r}-\mathbf{R}_{\mathbf{a}}\right) V_{1 b} \Phi_{1}\left(\mathbf{r}-\mathbf{R}_{\mathbf{b}}\right) d^{3} r \\
& \alpha_{12}=\int \Phi_{1}^{*}\left(\mathbf{r}-\mathbf{R}_{\mathbf{a}}\right) V_{2 s} \Phi_{2}\left(\mathbf{r}-\mathbf{R}_{\mathbf{s}}\right) d^{3} r \\
& \alpha_{21}=\int \Phi_{2}^{*}\left(\mathbf{r}-\mathbf{R}_{\mathrm{s}}\right) V_{1 a} \Phi_{1}\left(\mathbf{r}-\mathbf{R}_{\mathbf{a}}\right) d^{3} r \\
& \alpha_{2}=\int \Phi_{2}^{*}\left(\mathbf{r}-\mathbf{R}_{\mathrm{s}}\right) V_{2 t} \Phi_{2}\left(\mathbf{r}-\mathbf{R}_{\mathrm{t}}\right) d^{3} r \\
& H=\left(P^{2} / 2 m\right)+\sum_{\mathbf{a}} U_{1}\left(\mathbf{r}-\mathbf{R}_{\mathbf{a}}\right)+\sum_{s} U_{2}\left(\mathbf{r}-\mathbf{R}_{\mathbf{s}}\right) \\
& V_{1 b}=\sum_{c \neq b} U_{1}\left(\mathbf{r}-\mathbf{R}_{\mathbf{c}}\right)+\sum_{\mathbf{v}} U_{2}\left(\mathbf{r}-\mathbf{R}_{\mathbf{v}}\right) \\
& V_{2 s}=\sum_{c} U_{1}\left(\mathbf{r}-\mathbf{R}_{\mathbf{c}}\right)+\sum_{v \neq s} U_{2}\left(\mathbf{r}-\mathbf{R}_{\mathbf{v}}\right)
\end{aligned}
$$

We note that $\alpha_{12}=\alpha_{21} \equiv \beta$.

This follows from (4) and the relations that $U_{1}\left(\mathbf{r}-\mathbf{R}_{\mathbf{a}}\right) \Phi_{1}\left(\mathbf{r}-\mathbf{R}_{\mathbf{a}}\right)=\left(\varepsilon_{1}-p^{2} / 2 m\right) \Phi_{1}\left(\mathbf{r}-\mathbf{R}_{\mathbf{a}}\right)$ and $U_{2}\left(\mathbf{r}-\mathbf{R}_{\mathbf{s}}\right) \varphi_{2}\left(\mathbf{r}-\mathbf{R}_{\mathbf{s}}\right)=\left(\varepsilon_{2}-p^{2 / 2 m}\right) \Phi_{2}\left(\mathbf{r}-\mathbf{R}_{\mathbf{s}}\right)$.

In what follows we shall make the usual TBA simplifying assumption that $\alpha=0$ unless the wave functions are nearest neighbors and the $V$ is that belonging to one of the atomic wave functions. In addition, to simplify the mathematics even further, we will make the assumption that both $\phi_{1}$ and $\phi_{2}$ are $s$-like which makes the $\alpha$ s constant, independent of relative orientations between nearest neighbors. With these approximations equation (5) becomes

$$
\varepsilon_{k}=\varepsilon_{1}^{\prime}+\left(\varepsilon_{21}^{\prime} b b^{*}+\alpha_{1} B_{1}+b^{*} \beta B_{12}+b \beta B_{21}+b b^{*} \alpha_{2} B_{2}\right)\left(1+b b^{*}\right)^{-1} \text {. }
$$


where

$$
\begin{aligned}
& \varepsilon_{21} \dot{i}=\varepsilon_{2}{ }^{\prime}-\varepsilon_{1}^{\prime} \\
& B_{1}=\sum_{b}^{\prime} e^{i k \cdot\left(R_{a}-R_{b}\right)} \\
& B_{12}=B_{21}=\sum_{s}^{\prime} e^{i k \cdot\left(R_{a}-R_{s}\right)} \\
& B_{2}=\sum_{t}^{\prime} e^{i k \cdot\left(R_{s}-R_{t}\right)}
\end{aligned}
$$

The prime on the summation sign for the $B^{\prime} \mathbf{s}$ denotes that the sum is over the appropriate nearest neighbors only. $B_{12}=B_{21}$ by symmetry in an ordered 50-50 alloy, but not for other cases.

Minimizing $\varepsilon_{k}$ with respect to variations in $b$ or $b^{*}$ gives the two solutions

$$
b=\delta^{\prime} \pm\left[\left(\delta^{\prime}\right)^{2}+1\right]^{1 / 2} \text {. }
$$

where $\delta^{\prime}=\left(\varepsilon_{2} \dot{i}+\alpha_{2} B_{2}-\alpha_{1} B_{1}\right) / 2 \beta B_{12}$.

We see that in general $\delta^{\prime} \neq 0$ and thus $|b| \neq 1$. This means that the charges deposited on the two types of atoms differ from each other. Thus the charging effects that were discussed in the previous section have to be considered. The $\varepsilon_{21}^{\prime}$ and the $\alpha^{\prime} s$ themselves depend on the charging effects. Since they enter into the determination of $b$ and thus into how much charging is present, the effects of charging have to be estimated in a self-consistent manner. We defer the consideration of this problem till later on in this section.

The energy corresponding to the $b$ of (8) is

$$
\varepsilon_{k}=\varepsilon_{1}^{\prime}+\alpha_{1} B_{1}+\beta b B_{12}
$$

At the zero of $B_{12}, b$ varies from zero to $\infty$. This variation introduces a band gap of width $\Delta \varepsilon$ in the $\varepsilon_{k}$ spectrum

$$
\Delta \varepsilon=\varepsilon_{2} \dot{i}+\alpha_{2} B_{2}\left(\mathbf{k}_{0}\right)-\alpha_{1} B_{1}\left(\mathbf{k}_{0}\right)
$$

where $k_{0}$ is defined by the equation

$$
B_{12}\left(\mathbf{k}_{0}\right)=0
$$

Consider for example an ordered alloy with the cesium chloride structure where the atoms are ordered on a body-centered cubic lattice as shown in Fig. 1. In this case

$$
\begin{aligned}
& B_{1}=B_{2}=0 \\
& B_{12}=8 \cos \left(k_{x} a\right) \cos \left(k_{y} a\right) \cos \left(k_{z} a\right)
\end{aligned}
$$

where $k=\left(k_{x}, k_{y}, k_{z}\right)$ and $a$ is the length of a side of the cube in the Cecl structure. 


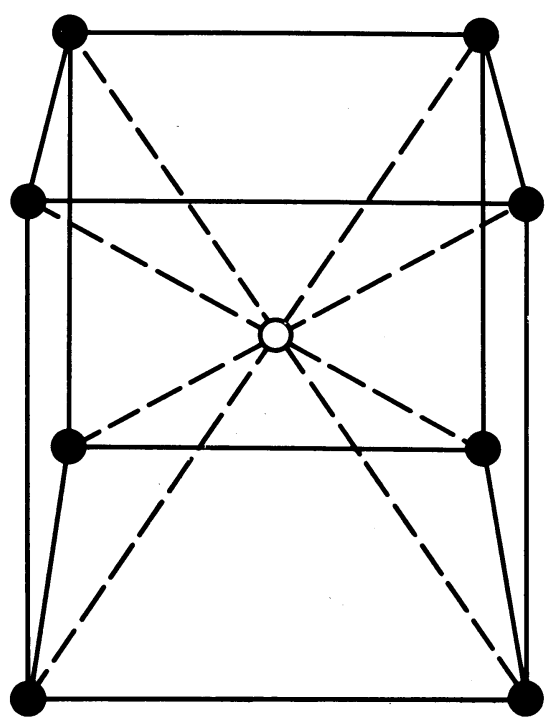

FIGURE 1

The cesium chloride crystal structure.

Figure 2 shows in a schematic manner the variation of the $\varepsilon_{k}$ curves and the density of states $\rho(\varepsilon)$ as a function of the parameter

$$
\delta_{0}=\varepsilon_{2} i / \Delta
$$

where $\Delta$ is the width of band below the gap. When $\delta_{0}$ is small, the density of states closely approximates that of the pure metal $\left(\delta_{0}=0\right)$ except near the center of the band. A band gap is introduced and the density of states is enhanced on either side of the gap in such a way that the enhancement just compensates for the gap. Away from the gap the change in the $\varepsilon_{k}$ and $\rho(\varepsilon)$ curves is of order $\delta_{0}{ }^{2}$ and is thus small for small $\delta_{0}$. When $\delta_{0}$ is large the band is effectively split into two parts, one band corresponding to the wave function concentrating on type one atoms to the exclusion of type two atoms, the other band corresponding to the reverse. Because there are assumed to be no spin dependent terms in the Hamiltonian, when spin is included the number of wave functions is doubled. Thus, for each value of $\mathbf{k}$ there are four wave functions since there are two possible values of $b$. Since there are $N$ independent values of $\mathbf{k}$ where $N$ is the number of unit cells in the alloy, there are a total of $4 N$ wave functions or 2 per atom as there should be.

We now try to estimate $\varepsilon_{21}^{\prime}$ for our model. To do this we need an expression for the amount of charging as a function of $\varepsilon_{21}^{\prime}$. The excess charge on type 2 atoms over the average we will denote by $\Delta \rho_{2}$. This is given by the expression

$$
\frac{\Delta \rho_{2}}{e}=\frac{2}{(2 \pi)^{3} N} \int\left(\frac{b^{2}}{1+b^{2}}-\frac{1}{2}\right) d^{3} k .
$$

Since, as can be seen from equations (3), (9), and (11), $b$ is only a function of $\varepsilon_{k}$, we can integrate (12) over a constant energy surface and obtain 


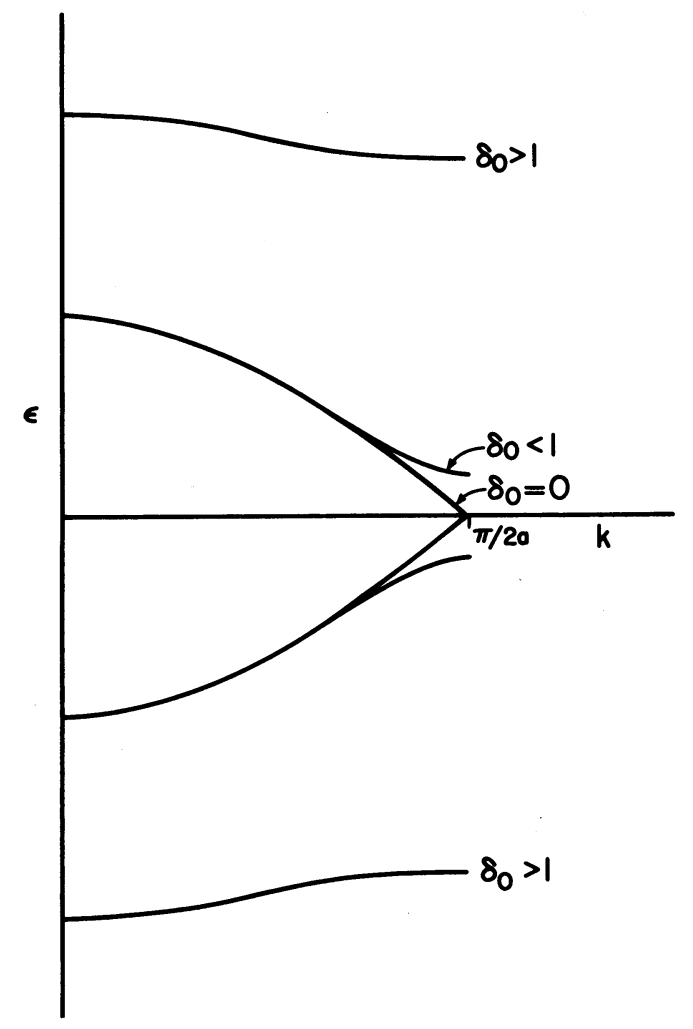

FIGURE $2 a$

The energy versus $k$ relation for the ordered binary alloy as a function of $\delta_{0}=\varepsilon_{21}^{\prime} / \Delta_{\text {. }}$ The result for the pure metal corresponds to $\delta_{0}=0$. When $\delta_{0}$ is small the result closely approximate the pure metal except for the gap in the middle.

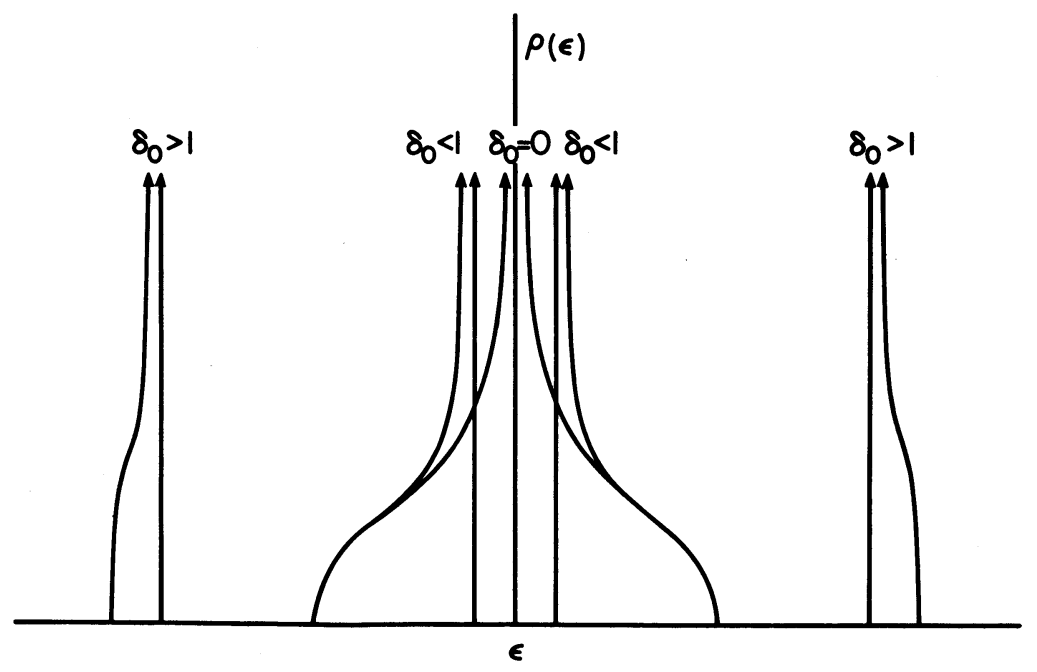

FIGURE 26

A sketch of the density of states curve for the ordered binary alloy as a function of $\delta_{0}$. The result for the pure metal corresponds to $\delta_{0}=0$. When $\delta_{0}$ is small the results closely approximate the pure metal except near the gap where there is an increase just equal to the deficit in the gap. 


$$
\frac{\Delta \rho_{2}}{e}=\frac{1}{2 N} \int_{E_{\text {m in }}}^{E_{F}}\left(\frac{b^{2}(E)-1}{b^{2}(E)+1}\right) \rho(E) d E
$$

where $e$ is the negative electronic charge, $E_{F}$ is the Fermi energy, $E_{\mathrm{min}}$ is the lowest energy in the band, and $\rho\left(\varepsilon_{k}\right)$ is the density of states per unit energy given by

$$
\rho(\varepsilon)=\frac{2}{(2 \pi)^{3}} \int \frac{d^{2} k}{\left|\nabla \varepsilon_{k}\right|}
$$

the integration being over a constant energy surface $\varepsilon_{k}$. In this discussion and throughout the paper it will be assumed that the alloy is at absolute zero temperature. Using equation (8) and (9), we can write the expression for $\Delta \rho_{2} / e$ as

$$
\frac{\Delta \rho_{2}}{e}=\frac{\varepsilon_{21}^{\prime}}{4 N} \int_{E_{\text {min }}}^{E_{F}} \frac{\rho(E) d E}{E-1 / 2\left(\varepsilon_{1}^{\prime}+\varepsilon_{2}^{\prime}\right)} .
$$

We see from $\left(12^{\prime}\right)$ that for a given $\varepsilon_{21}^{\prime}$ the maximum $\Delta \rho_{2}$ occurs when the band is half filled. The contribution to $\Delta p_{2}$ from above the gap is of opposite sign but equal in magnitude from below. When both the bands below and above the gap are completely filled, $\Delta \rho_{2}=0$ as it must by the Pauli exclusion principle.

First consider the case where both types of atoms contribute one TBA electron each. Then $E_{F}=\frac{\varepsilon_{1}^{\prime}+\varepsilon_{2}^{\prime}}{2}$ and using a mean value of the denominator the integral in (12') can be written as

$$
\frac{\Delta \rho_{2}}{e}=\frac{-\varepsilon_{21}^{\prime}}{2 f \Delta+\varepsilon_{21}^{\prime}}=\frac{-\delta_{0}}{2 f+\delta_{0}}
$$

where

$$
\varepsilon_{21}^{\prime}>0, f<1 \text {, and } \Delta=\varepsilon_{1}^{\prime}-E_{\text {in }} \text {. }
$$

From the form of the $\rho(E)$ curves for body-centered cubic, simple cubic and face-centered cubic lattices, which are all peaked at $\varepsilon=\varepsilon_{1}{ }^{\prime}, f$ is a number much less than one, and does not depend very greatly on $\delta_{0}$, though it does vary somewhat among different crystal structures. A reasonable value for $f$ is about $1 / 10$.

It remains to estimate how $\varepsilon_{1}^{\prime}$ and $\varepsilon_{2}^{\prime}$ vary as a function of $\Delta p$. This is quite a complicated problem when considered in detail. The excess charge on an atom changes the potential seen by an electron from that in the pure metal. Just how this charge changes the potential depends on various effects such as correlation between the electron and other electrons in the vicinity. This problem is considered in more detail in section 6. For our purposes, in this section, we will use a phenomenological argument. Regardless of the details of how the excess charge affects the potential, we expect, at least for small $\Delta p$, that the changes in $\varepsilon_{1}^{\prime}$ and $\varepsilon_{2}{ }^{\prime}$ should be proportional to $\Delta \rho$. Call the constant of proportionality $W$. Thus we can write

$$
\begin{aligned}
& \varepsilon_{1}{ }^{\prime}=\varepsilon_{1} 0+W_{1} \Delta \rho_{1} / e \\
& \varepsilon_{2}{ }^{\prime}=\varepsilon_{2} 0+W_{2} \Delta \rho_{2} / e
\end{aligned}
$$


where $\varepsilon_{1}{ }^{0}$ and $\varepsilon_{2}{ }^{0}$ are the values of $\varepsilon_{1}{ }^{\prime}$ and $\varepsilon_{2}{ }^{\prime}$, respectively, when $\Delta p=0$. From conservation of charge we have

$$
\Delta p_{1}=-\Delta p_{2}
$$

and inserting (13) and (14) into $\left(12^{\prime}\right)$ we find for $\Delta p$ and $\delta_{0}$ the expression

$$
\begin{gathered}
\delta_{0}=\frac{\varepsilon_{21} 0-2 f \Delta-W_{1}-W_{2}}{2 \Delta}+\left[\left(\frac{\varepsilon_{21} 0-2 f \Delta-W_{1}-W_{2}}{2 \Delta}\right)^{2}+\frac{2 f \varepsilon_{21} 0^{-}}{\Delta}\right]^{1 / 2} \\
\frac{\Delta \rho_{2}}{e}=-\frac{\left(\varepsilon_{21} 0+2 f \Delta+W_{1}+W_{2}\right)}{2\left(W_{1}+W_{2}\right)}+\left[\left(\frac{\varepsilon_{21} 0-2 f \Delta-W_{1}-W_{2}}{2\left(W_{1}+W_{2}\right)}\right)^{2}+\frac{2 f \varepsilon_{21}{ }^{0} \Delta}{\left(W_{1}+W_{2}\right)^{2}}\right]^{1 / 2}
\end{gathered}
$$

It is instructive to estimate numerically the charging effect and $\delta_{0}$. The parameters that must be assigned numerical values are; (a) $W_{1}$ and $W_{2}$, which indicate how the atomic energy of an atom charges with deviations from charge neutrality; (b) $\varepsilon_{21}{ }^{0}$, the difference in the atomic energies of the two atom types under charge neutrality conditions; (c) $\Delta$, the bandwidth of the alloy from the gap in the middle to the bottom of the band; and (d) $f$, the fraction of $\Delta$ that enters in calculating the deviations from charge neutrality (equation $12^{\prime \prime}$ ). We have previously estimated a numerical value for $f$ of $1 / 10$. This together with some typical values of the other parameters as discussed in more detail in section 6 are

$$
W_{1}=W_{2}=3 \mathrm{eV}, \varepsilon_{21} 0=1 \mathrm{eV}, \Delta=2.5 \mathrm{eV}, f=0.1 \text {. }
$$

With these numbers we find from equations (13) and (15) that

$$
\delta_{0} \approx 0.036, \Delta \rho_{2} / e \approx-0.15, \varepsilon_{21} \approx 0.09 \mathrm{eV}
$$

The charging effects are quite evident. They have decreased the gap in the middle of the band by a factor of eleven, deposited an excess charge of $0.15 e$ on type 1 atoms, and depleted an equal charge from the type 2 atoms. The band structure is close to that of a single constituent, except for the gap in the middle.

As a second example we let type one atoms contribute two TBA electrons each and type two atoms contribute one. This corresponds to alloying atoms of different valency. In this case the lower band is completely filled and the upper is half filled. Using similar reasoning, the expression for $\Delta \rho_{2} / e$, the excess above the average value of 1.5 , can be written approximately

$$
\frac{\Delta \rho_{2}}{e} \approx \frac{-\delta_{0}}{4 g+2 \delta_{0}}
$$

where $g \Delta$ is a bit greater than the width of the filled part of the top band. A reasonable value for $g$ is $1 / 3$. In this case of unequal valence constituents it is necessary to distinguish between two different charge deviations. The $\Delta \rho_{2}$ defined in (12"') is the charge deviation from an equal charze distribution on the two types of atoms. However, the factor that multiplies $W$ and produces changes in $\varepsilon^{\prime}$ is the deviation from charge neutrality of the atom which is not now $\Delta \rho_{2}$. Calling this deviation from charge neutrality $\sigma$ we can write the expression for $\varepsilon^{\prime}$

$$
\begin{aligned}
& \varepsilon_{1}^{\prime}=\varepsilon_{1} 0+W_{1} \sigma_{1} \\
& \varepsilon_{2}{ }^{\prime}=\varepsilon_{2} 0+W_{2} \sigma_{2}
\end{aligned}
$$




$$
\begin{array}{lll}
\text { where } & \sigma_{1}=\left(\Delta \rho_{1} / e\right)-0.5 \\
\text { and } & & \sigma_{2}=\left(\Delta \rho_{2} / e\right)+0.5 .
\end{array}
$$

Using the same values of the parameters as in (16) we find that

$$
\delta_{0} \approx 1.1, \quad \sigma_{2} \approx 0.3, \quad \varepsilon_{21}^{\prime} \approx 2.8 \mathrm{eV} .
$$

In this case the effect of charging is opposite from that of the preceding case. The type 2 atoms have negative $\Delta \rho_{2} / e$ as before but the net charge $\sigma_{2} e$ on the atom is $+.3 e$ which increases $\varepsilon_{21}$ ' above $\varepsilon_{21}{ }^{0}$. The gap in the middle of the band is now increased by charging and the band structure of the alloy is quite different from that of the single constituent case. This is typical result for an alloy with quite different valency constituents.

\section{Bloch Limit Alloy}

In this section we consider a binary disordered alloy. The spacing between the atomic energy levels in the solid is assumed to be very small; i.e., $\delta_{0} \ll 1$. From results of the last section we expect in this case that the energy levels and the wave functions will closely approximate those for a single constituent metal. We therefore, as a first approxination, assume a Bloch type wave function of the form

$$
\psi_{k}=N^{-1 / 2}\left(\sum_{a} e^{i \mathbf{k} \cdot \mathbf{R}_{\mathbf{a}}} \phi_{1}\left(\mathbf{r}-\mathbf{R}_{\mathbf{a}}\right)+\sum_{s} e^{i \mathbf{k} \cdot \mathbf{R}_{\mathbf{S}}} \phi_{2}\left(\mathbf{r}-\mathbf{R}_{\mathbf{s}}\right)\right)
$$

There are $N_{1}$ number of atoms of tyoe 1 with an atomic wave function $\phi_{1}$ and correspondingly for type 2 atoms. As in the previous section the letters of the first part of the alphabet indicate points on the lattice where type 1 atoms are situated and conversely for type 2. The middle portion is used when summing over both types 1 and 2 . Our model will be idealized, in what follows, by assuming that the positions of type 1 and 2 atoms are on a perfectly periodic array but randomly distributed on the array. We thus neglect distortion of the lattice and short and long range correlations between the different types of atoms. We assume the usual relations of the TBA given in (4) and use the same notation and approximations used in the previous section.

The Hamiltonian for this problem is

$$
H=\left(P^{2} / 2 m\right)+\sum_{a} U_{1}\left(\mathbf{r}-\mathbf{R}_{\mathbf{a}}\right)+\sum_{s} U_{2}\left(\mathbf{r}-\mathbf{R}_{\mathbf{s}}\right) .
$$

The average of $H$ over $\psi_{k}$ is given by

$$
\varepsilon_{k}=\left(N_{1} / N\right) \varepsilon_{1}(\mathbf{k})+\left(N_{2} / N\right) \varepsilon_{2}(\mathbf{k})+2\left(N_{1} N_{2} / N^{2}\right) \beta B(\mathbf{k})
$$

where

$$
\begin{aligned}
& \varepsilon_{1}(\mathbf{k})=\varepsilon_{1}+\left(N_{1} / N\right) \alpha_{1} B(\mathbf{k}) \\
& \varepsilon_{2}(\mathbf{k})=\varepsilon_{2}+\left(N_{2} / N\right) \alpha_{2} B(\mathbf{k})
\end{aligned}
$$




$$
\begin{gathered}
\varepsilon_{k}=\left(\int \psi_{k}^{*} H \psi_{k} d^{3} r\right)\left(\int \psi_{k}^{*} \psi_{k} d^{3} r\right)^{-1} \\
N=N_{1}+N_{2} \\
B(\mathbf{k})=\sum_{n} e^{i \mathbf{k} \cdot\left(\mathbf{R}_{\mathbf{m}}-\mathbf{R}_{\mathbf{n}}\right)} .
\end{gathered}
$$

As in the previous section, $\Sigma_{n}^{\prime}$ means the sum over the appropriate nearest neighbors to the site $\mathbf{R}_{\mathrm{m}}$, in this case all nearest neighbors since the middle of the alphabet is used to denote the site. To analyze these results further we note that the atomic wave function $\Phi_{1}$ and $\Phi_{2}$ can be chosen to be real. We also expect that $\alpha_{1} \approx \alpha_{2} \approx \beta$ since $\varepsilon_{12}{ }^{\prime}<1$. Much of the algebra simplifies without any qualitative change in results if $\alpha_{1}=\alpha_{2}=\beta=\alpha$. We will therefore assume these equalities for the rest of this section. Under this assumption $\varepsilon_{k}$ becomes

$$
\varepsilon_{k}=\left(N_{1} \varepsilon_{1}^{\prime}+N_{2} \varepsilon_{2}^{\prime}\right) / N+\alpha B(\mathbf{k}) .
$$

$B(\mathbf{k})$ for face centered cubic, body centered cubic and simple cubic lattices are given by, respectively,

$$
\begin{gathered}
B(\mathbf{k})=4\left[\cos \left(k_{x} a\right) \cos \left(k_{y} a\right)+\cos \left(k_{x} a\right) \cos \left(k_{z} a\right)+\cos \left(k_{y} a\right) \cos \left(k_{z} a\right)\right] \\
B(\mathbf{k})=8 \cos \left(k_{x} a\right) \cos \left(k_{y} a\right) \cos \left(k_{z} a\right) \\
B(\mathbf{k})=2\left[\cos \left(k_{x} a\right)+\cos \left(k_{y} a\right)+\cos \left(k_{z} a\right)\right] .
\end{gathered}
$$

The off diagonal matrix elements of the Hamiltonian are

$$
\begin{gathered}
H_{k l}=\left(\varepsilon_{1} \cdot / N\right) \sum_{a} e^{i(1-\mathbf{k}) \cdot \mathbf{R}_{\mathbf{a}}}+\left(\varepsilon_{2} \cdot / N\right) \sum_{s} e^{i(1-\mathbf{k}) \cdot \mathbf{R}_{\mathbf{s}}} \\
=\left(\varepsilon_{2} i / N\right) G(\mathbf{l}, \mathbf{k})
\end{gathered}
$$

where $G(\mathbf{l}, \mathbf{k})=\sum_{s} e^{i(1-\mathbf{k}) \cdot \mathbf{R}_{\mathbf{s}}}$. be

Since $\delta_{0}<<1$ the $H_{k l}$ are small and we find the wave function to first order in $\varepsilon_{21}^{\prime}$ to

$$
\mathrm{X}_{k}=\psi_{k}+\sum_{l} \frac{H_{l k} \psi_{l}}{\varepsilon_{k}-\varepsilon_{l}}
$$

These $X_{k}$ are scattered by $H_{k l}$ into other states of equal energy and the true eigenstates are linear combinations of the $x_{k}$ of equal energy. However, the scattering produces a width to the energy levels of the $x_{k}$ which is of the order of $\delta_{0}{ }^{2} \Delta$. Since we will calculate all quantities only to first order in $\delta_{0}$ we can neglect the effects of scattering.

The energy of $x_{k}$ is easily calculated and to first order in $\delta_{0}$ it is the same as given in 
$\left(19^{\prime}\right)$. Thus, neither the shapes of the energy spectrum nor the density of states of the alloys are changed to first in $\delta_{0}$ from their values in the pure state $\left(\delta_{0}=0\right)$. However, there is a first order change in the average charge deposited on a given atom by $\chi_{k}$ as we can find by calculating

$$
\Delta \rho_{2}(\mathbf{k}) / e=N_{2}-1 \sum_{\mathbf{s}}\left|\int \varphi_{2} *\left(\mathbf{r}-\mathbf{R}_{\mathbf{s}}\right) \chi_{k} d^{3} r\right|^{2}-N^{-1}
$$

Using (22), (21), and the orthogonality of the $\Phi_{1}$ we find

$$
\Delta \rho_{2}(\mathbf{k}) / e=\frac{2 E_{21}^{\prime}}{N_{2} N^{2}} \sum_{l} \frac{|G(\mathbf{l}, \mathbf{k})|^{2}}{E_{k}-E_{l}}
$$

This is the result for a given arrangement of the two types of atoms. Because of the large number of atoms involved, the average over the possible distribution of constituents describes the properties of the alloy. When averaged over all distributions of constituents, assuming a random distribution

$$
\overline{|G(\mathbf{l}, \mathbf{k})|^{2}}=N_{1} N_{2} / N
$$

and (24) becomes

$$
\Delta \rho_{2}(k) / e=2 \varepsilon_{2} i \frac{N_{1}}{N^{2}} P \int_{-\infty}^{\infty} \frac{\rho_{0}(\varepsilon) d \varepsilon}{\varepsilon_{k}-\varepsilon} .
$$

Here the sum in (24) is replaced by an integral by introducing the density of states

$$
\rho_{0}\left(\varepsilon_{k}\right)=\frac{2}{(2 \pi)^{3} V} \int \frac{d s}{\left|\nabla_{k} \varepsilon_{k}\right|} .
$$

The integral in (26) is over the constant energy surface in $\mathbf{k}$-space at $\varepsilon_{k}$, and $V$ is the volume of the solid. The principal value of the integral in $\left(24^{\prime}\right)$ is to be taken as indicated by the $P$ just before the integral. In a similar manner we find that

$$
\Delta \rho_{1}(\mathbf{k}) / e=-2 \varepsilon_{2} i \frac{N_{2}}{N^{2}} P \int \frac{\rho_{0}(\varepsilon) d \varepsilon}{\varepsilon_{k}-\varepsilon} .
$$

Just as in the previous section for the ordered alloy, the disordered alloy also shows a charging effect given by $\left(24^{\prime}\right)$ and $\left(24^{\prime \prime}\right)$. When $\varepsilon_{k}$ is below the middle of the band $\Delta p_{1}(\mathbf{k}) / e$ has the same sign as $\varepsilon_{21}^{\prime}$ and vice versa for $\varepsilon_{k}$ above the middle of the band. This sign for $\Delta p_{1}(\mathbf{k}) / e$ is the same as found for the ordered alloy and is also the same as expected from the general considerations given in Section 2. The constituent with the lowest atomic energy, which we have by convention called 1 , has more electronic charge deposited on it.by the lowest energy states in order to minimize the energy.

In a completely filled band no charging can exist as can be seen by summing $\Delta \rho(\mathbf{k})$ over all states. From $\left(24^{\prime}\right)$ or $\left(24^{\prime \prime}\right)$ the integral of $\Delta p(\mathbf{k})$ over all states is zero because of the occurrence of the following term 


$$
P \int_{-\infty}^{\infty} \int_{-\infty}^{\infty} \frac{\rho_{0}\left(\varepsilon^{\prime}\right) \rho_{0}(\varepsilon) d \varepsilon^{\prime} d \varepsilon}{\varepsilon^{\prime}-\varepsilon}=0
$$

which is zero since the integrand is odd in the interchange of $\varepsilon$ and $\varepsilon^{\prime}$. This disappearance of a charging effect in a full band is a general result not limited to first order perturbation theory. It is a consequence of the completeness of the wave functions and can be seen as follows. Let the exact wave functions of the alloy be denoted by $\Omega_{m}$, a complete orthonormal set The $\Phi_{i}\left(\mathbf{r}-\mathbf{R}_{\mathbf{n}}\right)$ also are a complete orthonormal set for the alloy as indicated by equation (4) The $\Phi_{i}$ can be expanded in terms of the $\Omega_{m}$ and are given by

$$
\phi_{i}=\sum_{m} \Omega_{m} \int \varphi_{i} * \Omega_{m} d^{3} r
$$

Calculating the absolute square of both sides of (28) we obtain

$$
1=\sum_{m}\left|\int \phi_{i} * \Omega_{m} d^{3} r\right|^{2}
$$

But the right-hand side of (29) is the total number of electrons in state $\Phi_{i}$ on the atom at the lattice site $\mathbf{R}_{\mathbf{n}}$ for a full band. Thus, all sites have one electron per state when the band is full and no charging occurs.

It is of interest to compare the ordered and disordered alloys in the limit $\delta_{0} \ll 1$. The states of the ordered alloy over most of the band deposit a charge of order of $\delta_{0}$; but within the region of energy $\varepsilon^{\prime}{ }_{21}$ from $\varepsilon_{1}{ }^{\prime}$ and $\varepsilon_{2}{ }^{\prime}$, a large amount of charging occurs. This large charging produces the gap between the energies $\varepsilon_{1}^{\prime}$ and $\varepsilon_{2}{ }^{\prime}$. No such region of large charging occurs for the disordered alloy. The charging is of order $\delta_{0}$ throughout the whole band. This difference in behavior occurs because of the properties of the $H_{k l}$ of (21). In the disordered alloy $G(\mathbf{l}, \mathbf{k})$ is a random function and has a root mean square deviation from zero, its average value, of the order of $\sqrt{N}$ as shown by (25). Thus, $H_{k l}$ in the disordered alloy is of the order of $N^{-1 / 2}$ for all values. In contrast, in the ordered alloy, $H_{k l}$ is zero, except when $(\mathbf{k}-\mathbf{l})$ is close to a reciprocal lattice vector of the ordered alloy when $H_{k l}$ is of order one. This large value of $H_{k l}$ in the ordered case produces the gap. No such dominance of a particular value of $H_{k l}$ occurs in the disordered alloy and no gap is produced. In fact, the density of states of the disordered alloy closely approximates that of the pure metal $\left(\delta_{0}=0\right)$ differing only in order $\delta_{0}{ }^{2}$. This is illustrated in Fig. 3 where the density of states of the Block limit alloy $\delta_{0}<<1$ is compared with that of the pure metal, $\delta_{0}=0$. As can be seen, the only appreciable difference between the alloy and the pure metal is that the sharp structure at the middle of the band is somewhat smeared out in the alloy. Such an alloy satisfies the criterion for the validity of the rigid band approximation.

\section{Atomic Limit Alloy}

In this section we discuss the limit $\delta_{0}=\frac{\varepsilon_{2} \dot{1}}{\Delta} \gg 1$ for a completely disordered binary alloy. This corresponds to the case when the atomic levels are separated by an amount much greater than the width of the bands. To guess what form the wave function will take in this case we note that the last two sections suggest that the electrons will be mainly on either one or the other type of atom. Also $\delta_{0} \gg 1$ can be interpreted as near the limit $a \rightarrow 0$. When $\alpha=0$ the wave function is rigorously the sum of atomic wave functions and the energy levels 


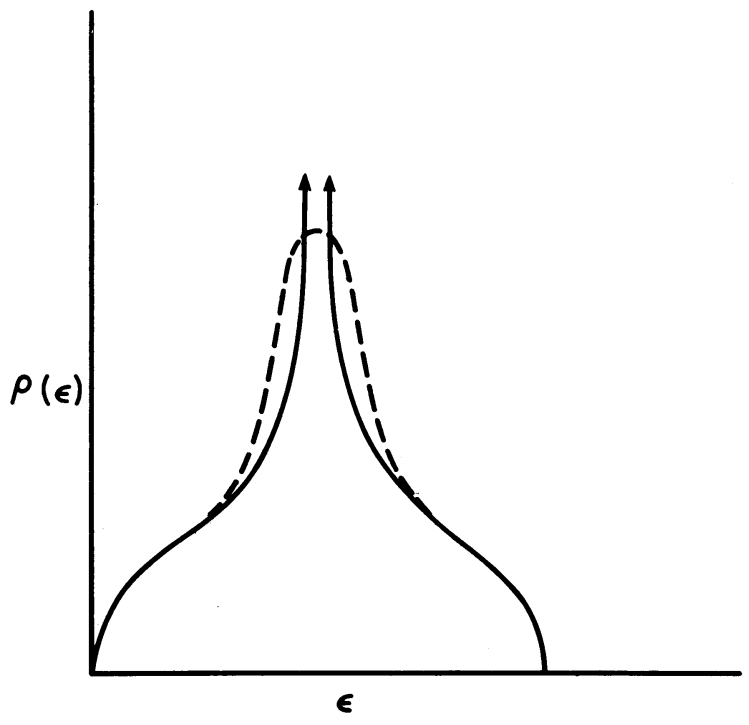

FIGURE 3

A sketch of the density of states curve for a pure metal (solid line) and a completely disordered alloy (dashed line) in the limit that $\delta_{0} \ll 1$. Both metals have a body-centered-cubic structure and the TBA electrons have $s-1$ ike symmetry.

The structures are quite similar except that the infinity in the pure metal is finite and somewhat wider in the alloy.

are just $\varepsilon_{1}$ and $\varepsilon_{2}$. For the energy $\varepsilon_{1}$ the wave functions are simply

$$
\psi_{k}=\sum_{a} a_{k a} \Phi_{1}\left(\mathbf{r}-\mathbf{R}_{\mathbf{a}}\right)
$$

where $a_{k a}$ are constants. In order to orthogonalize these wave functions we can set

$$
a_{k a}=e^{i \mathbf{k} \cdot \mathbf{X}_{\mathbf{a}}}
$$

where

$$
\mathbf{k} \cdot \mathbf{x}_{\mathbf{a}}=2 \pi \sum_{i}\left(n_{i a} l_{i}\right) / L_{i}, \quad(i=1,2,3)
$$

and $n_{i a}$ are integers, a given combination of which corresponds to the atom at point $\mathbf{R}_{\mathbf{2}}$. Also,

$$
\mathbf{k}=\frac{l_{1}}{L_{1}}, \frac{l_{2}}{L_{2}}, \frac{l_{3}}{L_{3}}
$$

the l's are integers and $L_{1} L_{2} L_{3}=N_{1}$.

The wave functions will be orthogonalized regardless of the $\mathbf{x}_{\mathbf{a}}$ that is associated with the atom at point $\mathbf{R}_{\mathbf{a}}$. However, in order to minimize the off-diagonal elements of the Hamiltonian when $\alpha \neq 0$, a special combination is chosen as follows. A fictitious lattice of points is superimposed on the real lattice. This fictititous lattice is chosen to have the same structure and fill the same volume as the real lattice but only has $N_{i}$ points instead of $N$. The type 1 atoms are associated with the closest point in the fictitious lattice consistent with keeping the same nearest type 1 neighbors and the same configuration as in the real lattice. This cannot be done exactly because of statistical fluctuations. A fraction of the atoms have to be "removed" 
from their closest point in the fictitious lattice and placed elsewhere in order to maximize the correspondence between the nearest neighbors in the two lattices. This number of "removed" atoms may be small and will be neglected. We will assume that all the nearest type 1 neighbors in the real lattice are also nearest type 1 neighbors in the fictititous lattice, though the reverse is not true. This is taken into account by multiplying the number of nearest neighbors in the fictitious lattice by $N_{\mathrm{l}} / N$, the probability that any nearest neighbor in the fictitious lattice is also one in the real lattice.

When the overlap $\alpha \neq 0$ but $\delta_{0} \gg 1$ we expect for energies near $\varepsilon_{1}$ the wave functions will have the form

$$
\left(N_{1}+\sum_{s} A_{k s} A_{k s}^{*}\right)^{1 / 2} \psi_{k}=\sum_{s} e^{i \mathbf{k} \cdot \mathbf{x}_{\mathbf{a}}} \varphi_{1}\left(\mathbf{r}-\mathbf{R}_{\mathbf{a}}\right)+\sum_{s} A_{k s} \Phi_{2}\left(\mathbf{r}-\mathbf{R}_{\mathbf{s}}\right)
$$

where $A_{k s}$ are small. For energies near $\varepsilon_{2}$ the wave functions will have the form

$$
\left(N_{2}+\sum_{a} A_{l a} A_{l a}^{*}\right)^{1 / 3} \phi_{l}=\sum_{a} A_{l a} \Phi_{1}\left(\mathbf{r}-\mathbf{R}_{\mathbf{a}}\right)+\sum_{s} e^{i \mathbf{l} \cdot \mathbf{x}_{\mathbf{s}}} \phi_{2}\left(\mathbf{r}-\mathbf{R}_{\mathbf{s}}\right)
$$

where the $\mathbf{x}_{\mathbf{s}}$ form a lattice of the same type as the real lattice and filling the same volume but containing $N_{2}$ points. The $A_{l a}$ are expected to be much less than one. The states in (33) and (34) are not orthogonal to one another, the overlap being of order $A$ and thus small. We will limit ourselves to zero order in $A$ so that we can neglect the non-orthogonality of the wave functions.

The energy is $E(k)=H_{k k}$,

$$
\begin{gathered}
\left(N_{1}+\sum_{s} A_{k s} A_{k s}^{*}\right) E(\mathbf{k})=N_{1} \varepsilon_{1}^{\prime}+\frac{N_{1}^{2}}{N} B(\mathbf{k}) \alpha_{1} \\
+\sum_{s} \sum_{a} \alpha_{12}\left(A_{k s} e^{-i \mathbf{k} \cdot \mathbf{x}_{\mathrm{a}}}+A_{k s}^{*} e^{i \mathbf{k} \cdot \mathbf{x}_{\mathbf{a}}}\right) \\
+\sum_{s} A_{k s A_{k s}^{*} \varepsilon_{2}}+\sum_{s} \sum_{t} \alpha_{2} A_{k s}^{*} A_{k t}
\end{gathered}
$$

where $B(\mathbf{k})=\sum_{a}^{\prime} e^{i \mathbf{k} \cdot\left(\mathbf{x}_{\mathbf{b}}-\mathbf{x}_{\mathbf{a}}\right)}$ and the sum is over nearest neighbors in the fictitious lattice. $B(\mathbf{k})$ is the same as the $B(\mathbf{k})$ defined in the previous section and its value for various types of structures is given in (20). Again, as in the previous section, the atomic wave functions $\Phi_{1}$ and $\Phi_{2}$ are both assumed to be spherically symmetric and real.

Differentiating $E(\mathbf{k})$ with respect to $A_{k}$ and setting it equal to zero gives

$$
A_{k s^{*}}=\left(\alpha_{12} / \varepsilon_{12}{ }^{\prime}\right) \sum_{a} e^{-i \mathbf{k} \cdot \mathbf{x}_{\mathrm{a}}}
$$

where the sum is over the lattice points with type 1 atoms which are nearest neighbors to point $\mathbf{R}_{\mathbf{s}}$ in the real lattice. This value for $A_{k s}{ }^{*}$ will be corrected by terms of order $A$ when orthogonalization is imposed. However, we do see fror (36) that $A_{k s}$ and thus $A$ is of order $1 / \delta_{0}$, : 
and is small, confirming our initial guess.

To zero order in $A$, equation (35) becomes

$$
E(\mathbf{k})=\varepsilon_{1}^{\prime}+\left(N_{1} / N\right) \alpha_{1} B(\mathbf{k}) .
$$

We see that alloying decreases the width of the band in direct proportion to the dilution of the type 1 atoms. However, the shape remains the same. The density of states of the alloy is calculated as follows. The density of states $\rho_{0}(E)$ of the pure solid of type 1 atoms $\left(V_{2}=0\right)$ is given by

$$
\rho_{0}(E)=\frac{2}{(2 \pi)^{3} \alpha_{1} V_{0}} \iint \frac{d s}{\left|\nabla_{k} B(k)\right|}
$$

where $V_{0}$ is the volume of the pure solid. The density of states of the alloy $\rho(E)$ is given by

$$
\rho(E)=\left(N V_{0} / N_{1} V\right) \rho_{0}(E)
$$

where $V$ is the volume of the alloy. Assuming that the alloying keeps the lattice constant the same, as is being assumed throughout, then adding $N_{2}$ type 2 atoms changes the volume from $V_{0}$ to

$$
V=\left(N / N_{1}\right) V_{0} \text {. }
$$

Thus we see that

$$
\rho(E)=\rho_{0}(E)
$$

and alloying does not change the density of states. Alloying has two effects which cancel one another. The width of the band is decreased because the overlap is smaller, but the number of type 1 atoms per unit volume also decreases, the combination of the two effects keeping the density of states fixed.

In the above discussion we have neglected the effect of scattering. To estimate this to zero order we calculate the transition rate by first evaluating

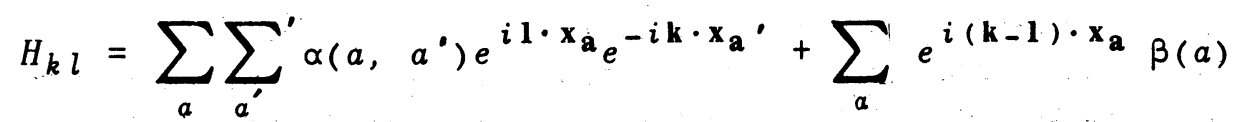

where the sum over $a^{\prime}$ is over nearest neighbors in the real lattice and

$$
\begin{gathered}
\beta(a)=\int\left[\sum_{a^{\prime} \neq a} U_{1}\left(\mathbf{r}-\mathbf{R}_{\mathbf{a}}^{\prime}\right)+\sum_{s} U_{2}\left(\mathbf{r}-\mathbf{R}_{\mathbf{s}}\right)\right] \varphi_{1} *\left(\mathbf{r}-\mathbf{R}_{\mathbf{a}}\right) \Phi_{1}\left(\mathbf{r}-\mathbf{R}_{\mathbf{a}}\right) d^{3} r \\
\alpha\left(a, a^{\prime}\right)=\int\left[\sum_{a^{\prime} \neq a} U_{1}\left(\mathbf{r}-\mathbf{R}_{\mathbf{a}}{ }^{\prime}\right)+\sum_{s} U_{2}\left(\mathbf{r}-\mathbf{R}_{\mathbf{S}}\right)\right] \varphi_{1} *\left(\mathbf{r}-\mathbf{R}_{\mathbf{a}}{ }^{\prime}\right) \Phi_{1}\left(\mathbf{r}-\mathbf{R}_{\mathbf{a}}\right) d^{3} r
\end{gathered}
$$

The quantities $\beta(a)$ and $\alpha\left(a, a^{\prime}\right)$ are random variables because the number of type 1 and type 2 neighbors will vary at each point $\mathbf{R}_{\mathrm{a}}$. In the Bloch limit discussed in the previous section the variation in $\beta(a)$ was neglected because it was assumed that since $\varepsilon_{1}{ }^{\prime} \approx \varepsilon_{2}{ }^{\prime}, U_{1}$ and $U_{2}$ are closely the same at points in the overlap region. We cannot expect this to be true in the atomic limit discussed here. The energy width $\Delta E$ produced by scattering is given by 


$$
\Delta E=2 \pi\left|H_{k l}\right|^{2} \rho(E)
$$

and we estimate that $\Delta E / 2 \Delta$, the energy width divided by the band width, is of order $N_{2} / N_{1}$. Thus if the dilution is not too great the scattering will not affect the shape of the band. However, when $N_{2} \approx N_{1}$, scattering will become important and we can expect that the shape will be appreciably changed by it.

The results for the band of the type 2 atoms can be obtained from the previous results by interchanging the subscripts 1 and 2. Thus the ratio of the energy width of a single state produced by scattering divided by the band width is of order $N_{1} / N_{2}$. In the case where scattering does not appreciably affect the properties of the band of type 1 atoms $\left(N_{2} / N_{1}<<1\right)$, the properties of the band of type 2 atoms is greatly affected by scattering and cannot be determined from the analysis here. This problem is that of the density of impurity states in dilute alloys where the impurity states are bound, and it has to be solved by different techniques than the ones employed in this paper. However, quantities such as the specific heat, magnetic susceptibility, etc., will depend only on the properties of the host band as long as it is not completely filled.

\section{Pseudoatomic Limit}

Finally we consider another case which formally gives the same results as the atomic limit. The TBA electron wave functions of the type 2 atoms have a smaller radius than the type 1 atoms. To be more precise we assume that $\alpha_{12}=\alpha_{21} \ll \alpha_{1}$ and $\alpha_{2} \ll \alpha_{1}$. Under these conditions we find, using the same procedure as employed in the atomic limit, that

$$
A_{k s} \approx\left(\alpha_{12} \sum_{a=s+1} e^{i k \cdot x_{a}}\right)\left(\varepsilon_{2} i-\alpha_{1} B(k) N_{1} / N\right)^{-1}
$$

The wave function is assumed to have the same form as given in (33) and $A_{k s}$ is assumed to be much less than unity. We see from (44) that $A_{k s}$ is much less than unity if the difference between the energy of the $k$-state in the type 1 band in the atomic limit (obtained by removing the type 2 atoms without disturbing the type 1 atoms) and $\varepsilon_{2}{ }^{\prime}$ is in absolute magnitude much greater than $\alpha_{12}$. When this occurs the properties of the alloy are exactly the same as of the type 1 band in the atomic limit.

\section{Application to Real Metals}

We discuss in this section how the models presented in the previous sections can be applied to real metals. The previous sections considered a model of the band structure of binary alloys in the TBA with $s$-type electrons. It is possible to extend the analysis to cover the case of $d$ bands. At first we neglect hybridization among the $d$ sub-bands. For each spin there are then 5 degenerate atomic $d$-states each having approximately equal values of $\varepsilon$. We can proceed with the analysis for each atomic $d$-state separately and obtain the same type of result as for $s$ states. Adding spin doubles the number of states as before. We also assume that for transition metal alloys the number of $s$-electrons per atom in the alloy is the mean of those in the two constituents, so there is no transfer of electrons from $s$ - to $d$-bands. 


\subsection{Estimate of Parameters}

As shown in Sections 3 and 4, when the parameter $\delta_{0} \ll 1$ the rigid band approximation is valid. For a disordered alloy this statement is true in the sense that the energy spectrum (see equation $\left(19^{\prime}\right)$ ) and the wave functions (see equation (22)) have a deviation of order $\delta_{0}^{2}$ and $\delta_{0}$, respectively, from those of a single constituent metal. The property of this single constituent metal is some average of the properties of the two elements in the alloy. This average depends, among other things on the charging effects which modify the shape of the atomic wave functions and thus the overlap integrals. As discussed in Section $3, \delta_{0}$ has to be determined selfconsistently. The magnitude of $\delta_{0}$ depends on charging effects and the charging effects themselves depend on $\delta_{0}$. This self-consistent calculation requires the knowledge of three parameters. These are: (1) the $\varepsilon^{0}$; (2) the $W$ introduced in equation (13); and (3) the relation between $\delta_{0}$ and $\Delta \rho$. We discuss how to estimate these parameters in what follows.

(1) The energy $\varepsilon^{0}$ has two parts to it. It is composed of an effective Hartree-Fock enerzy of the isolated atom plus an additional term produced by the potentials of the surrounding atoms adding to the isolated atomic potential. It is difficult to determine the effective HartreeFock energy of the isolated atom. This is defined as the energy required to remove an electron in the state of interest keeping all the other electron states unchanged. This energy cannot simply be obtained empirically from data on the excited states of atoms because in the real case when an electron is removed, the revaining electrons see a changed potential which changes their wave functions. Another way to determine $\varepsilon^{0}$ is to note that in the TBA $\varepsilon^{0}$ is just the average energy of the $d$-band [3]. The expected shape of the $d$-band can be estimated from the theoretical calculations of Wood [4] for iron. By using the measured values of the work function of transition metals which give the absolute position of the Fermi level, and assuning that Wood's calculation is a reasonable approximation to the shape of the $d$-band in all transition metals, the absolute position of the average energy of the $d$-band can be obtained. In this way it can be estimated for the transition metals that $\varepsilon^{0}$ decreases about $/ 2$ eV for each increase of one unit in the nuclear charge.

(2) The parameter $W$ is the constant of proportionality between $\sigma$, the deviation from neutrality of the average charge around an atom, and the atomic energy. From the virial theorem the corresponding change in the potential energy is twice as great. The parameter $W$, if the $s$ electrons had no screening effect, would have a value of the order $e^{2} / r_{D}$, where $r_{D}$ is the radius of the atomic orbital of a $d$-electron. This is about $10 \mathrm{eV}$. However, most recent workers on the origin of ferromagnetism have agreed that this quantity must be greatly cut down by screening by the $s$ electrons, though they differ about the amount of this reduction. A review of the different theories has been given by Mott [1]. In addition, correlation between the $d$ electrons themselves will decrease $W$. We shall adopt the point of view taken in Mott's review article, that $W$ is unlikely to be greater than half the band width. In fact we have been implicitly assuming this all along by using the one-electron approximation. If $W$ is greater than half the band width then, as Hubbard [5] shows, correlation effects are important and the oneelectron approximation is incorrect.

(3) The relationship between $\Delta p$ and $\delta_{0}$ for small $\delta_{0}$ is obtained with the aid of the fact that in a full band there is no charging present. When the Fermi energy $E_{F}$ is above or below the middle of the band the charging effect can be obtained, respectively, by either the negative of the integral $\Delta p(\mathbf{k})$ of equations $\left(24^{\prime}\right)$ and $\left(24^{\prime \prime}\right)$ from $E_{F}$ to the top of the band, or by the integral from the bottom of the band to $E_{F}$. When $\delta_{0}$ is large, the charging effect is obvious since the electronic states congregate on either one or the other type of atom as discussed in Section 5 . 


\subsection{Alloys with Non-transition Metals}

Alloys of $\mathrm{Ag}$ and $\mathrm{Au}$ with $\mathrm{Pd}$ and $\mathrm{Pt}$, respectively, have been used to obtain information on the density of states curve [6]. Although this is an alloy between a transition metal and a nontransition metal we can still apply our analysis to this case. The experimental and theoretical evidence is that the $d$-bands in $\mathrm{Ag}$ and $\mathrm{Au}$ are appreciably narrower than in the transition metals. The states near the Fermi level in the alloys under discussion satisfy the pseudoatomic limit discussed at the end of Section 5. The $d$-bands in $\mathrm{Ag}$ and $\mathrm{Au}$ remain filled and the density of states for small amounts of alloyed $\mathrm{Ag}$ and $\mathrm{Au}$ is the same as for pure $\mathrm{Pd}$ and Pt. However, when the number of $\mathrm{Ag}$ and $\mathrm{Au}$ atoms becomes comparable to that of $\mathrm{Pd}$ and $\mathrm{Pt}$, scattering effects should become important and change the shape of the density of states curve. Thus, the tail at the high energy end of the $d$-band of Pd and Pt found by this means is almost certainly not present in the pure metal. This discussion only applies to the $d$-bands in these alloys. To determine the distribution of the electrons between the $s$ - and $d$-bands requires an analysis of the $s$-bands in these same alloys. Such an analysis is not appropriate for this paper and will not be attempted here.

In the case of alloys of transition metals with other non-transition ones we can again describe what happens to the $d$-band though we cannot predict the distribution of the electrons between the $d$ - and $s$-bands because this depends also on details of the $s$-band of the alloy. When a transition metal is alloyed with a non-transition metal the situation is approximated by either the atomic limit or pseudoatomic limit discussed in Section 5 . In the case of the noble metals just described the pseudoatomic limit was appropriate. In the case of $\mathrm{Al}$, say, the atomic limit is more appropriate since the energy of any state that could be treated by the TBA is greatly removed from the energy of the $d$-bands in the transition metals. In any case, the result on the $d$-band of the alloy is the same. For small concentrations of the non-transition metal the $d$-band of the alloy has the same density of states as the pure transition metal, and when the concentration of the non-transition metal is comparable to that of the transition metal, scattering effects change the shape of the density of states curves.

\section{Summary and Discussion}

In this paper a theory of the electronic structure of alloys in the TBA has been presented. The problem of an ordered $50 \%-50 \%$ alloy is solved exactly in the TBA. Then are considered the cases of disordered alloys of arbitrary composition and in the limits where $\delta_{0}<<1$, the Bloch limit, $\delta_{0} \gg 1$, the atomic limit, and the pseudoatomic linit where the band width of the pure metal of one constituent is much less than that of the other. Here $\delta_{0}$ is the ratio between the difference of the atomic energies of the atoms and half the width $\Delta$ of the band. In the Bloch limit the alloy system has a single band structure which closely approximates that of a pure metal so that the rigid band approximation is valid. The pure metal that is approximated is some average of the two constituents and the averaging process depends on the relative concentration of the two constituents. The main mechanism that determines the averaging is the charging effect which changes the potential seen by a TBA electron and thus its wave function, especially in the important overlap region between neighbors which determines the shape of the band. In the atomic limit the alloy has two separate band structures. These bands are the same as obtained by removing one constituent from the alloy without disturbing the other. Neglecting scattering effects, the density of states of the alloy in the atomic limit is the same as the pure metal consisting of one or the other constituent, depending on which band the Fermi level is in. Alloying decreases the overlap between like atoms and narrows the band tending to increase the density of states. This is just cancelled by the increased total number of atoms for a given number of like atoms so that the specific heat per unit volume or mole remains fixed. 
Scattering effects in a band become important as the number of other atoms becomes of the order of the number of atoms that comprise the band. In the transition region between the Bloch limit and the atomic limit the shape of the density of states of the alloy is greatly modified from the pure metal case and measurements on the alloy cannot directly give information on the pure metal.

The important characteristic of the theory is that there are differing amounts of charge on the two types of atom. In the Bloch limit the difference in charge is of order $\delta_{0}$, while in the atomic and pseudoatomic limits practically all of the charge concentrates on one type of atom or the other. Except when the band or bands are completely full, there will be a difference in charge between the two constituents. This charging effect will change the relative atomic energies of the two constituents and their overlap integrals.

The magnitude of the charging effects and the value of $\delta_{0}$ have to be determined in a selfconsistent manner. The values of $\varepsilon_{21}{ }^{\prime}$ and $\Delta$ and thus $\delta_{0}$ depend on the charging effects while the difference of charge deposited on each atom depends on $\delta_{0}$ and the position of the Fermi level. When the Fermi level is near the top of the band of a Bloch limit alloy, a larger $\delta_{0}$ is required for a given net charge difference than when the Fermi ievel is near the middle of the band or bands. The middle of the band or bands is defined to be at the energy $\left(N_{1} \varepsilon_{1}{ }^{\prime}+N_{2} \varepsilon_{2}{ }^{\prime}\right) / N$.

When many body effects are neglected, the theory justifies the use of the rigid band model to calculate from specific heat measurements on PdAg and AuPt alloys the density of states curve for the $d$-band of the pure Pd and Pt. This is justified as long as the concentration of $\mathrm{Ag}$ and $\mathrm{Au}$ is not too great. At large concentrations of $\mathrm{Ag}$ and $\mathrm{Au}$ scattering effects will be important and are probably the cause of the tail found in the density of states curves at the top of the $d$-band in these alloys. Also, the distribution of the electrons between the $d$ - and $s$-bands is not given by the theory since this also depends on details of the $s$-band.

An important parameter that enters the theory is $W$ which is a measure of how much charging changes the value of $\varepsilon^{\prime}$. This parameter is closely related to the Coulomb effects envisaged by Wolff [7], Hubbard [5], Anderson [8], Van Vleck [9], and Kanamori [10] for problems different from the one considered here. The calculation of $W$ is a difficult many body problem which must include screening effects by the other $d$-electrons and the surrounding $s$-electrons in a strong atomic potential.

An important simplification made in this paper is the assumption that the total width of the $d$-band in all of the transition metals is approximately the same. If this is far from the truth then an important error has been introduced. As an example consider the case of two metals, 1 and 2. Metal 1 in the pure state has a $d$-band with ten times the width of that for metal 2 . Also let $\varepsilon_{1}^{\prime}=\varepsilon_{2}{ }^{\prime}$ in a $50 \%-50 \%$ alloy of the two metals. We know from our analysis of the pseudoatomic limit in Section 5 that, neglecting scattering effects, the total width of the band in the alloy will be half that of pure metal 1, and states near the top of the band will be depositing essentially all of their charge on the 1 atoms. When the band is completely full all atoms have the same charge, $10 d$-electrons. If the Fermi level is near the top of the band then the 2 atoms still practically have a charge of $10 e$ but 1 atoms will have less charge, since the states that are empty deposit charge essentially only on the 1 atoms. Following this argument further it is easy to see that there must be other states which preferentially deposit charge on the type 2 atoms. Thus we obtain important charging effects even when $\varepsilon_{1}{ }^{\prime}=\varepsilon_{2}{ }^{\prime}$ if $\alpha_{1}$ is quite different from $\alpha_{2}$. Figure 4 shows in a schematic manner the variation of the charging effect of a state as a function of energy and the density of states for this case.

It was assumed throughout that alloying produces no distortion of the lattice. Of course, distortion of the lattice does occur in practice. Offhand it does not appear that the amount 


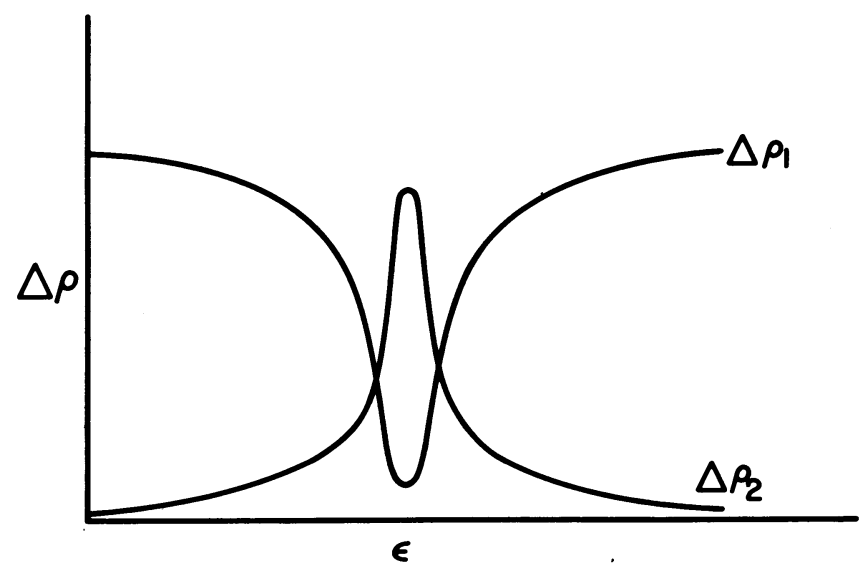

FIGURE $4 a$

A sketch of the charging effect of a electronic state as a function of energy for a disordered binary alloy where the two constituents have quite different band widths in the pure state. It is assumed that $\varepsilon_{1}^{\prime}=\varepsilon_{2}^{\prime}$ and type 2 atoms have the narrower bandwidth. The relative number of electrons deposited on type 1 atoms is shown by the curve marked $\Delta p_{1}$ and correspondingly for the curve marked $\Delta p_{i 2}$.

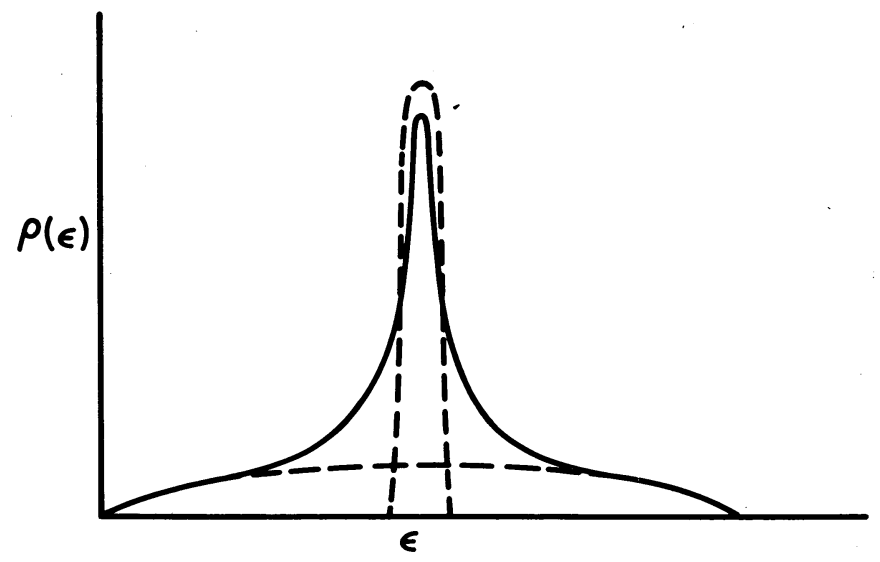

FIGURE 46

The density of states curve for the same alloy as shown in Fig. 4a. The dashed curves are the density of states curves of the pure metals contracted by a factor of 2 in the energy scale.

of distortion found in actual alloys will qualitatively change anything. However, quantitatively the overlap integrals and the potentials seen by an electron will be affected by distortion, which should also quantitatively effect the expressions for $\Delta p(k)$ and $E(k)$. 
Finally, for the transition metal alloys it was assumed that the number of $s$-electrons per atom in the alloy is just the average of those in the two pure metals. The number of $s$-electrons in the vicinity of an atom was allowed to vary in the alloy in order to give screening but no net transfer of electrons between the $s$ - and $d$-bands was allowed to occur. Probably not much transfer between the two bands does occur in alloys of transition metals but this is uncertain. It is unclear if any important qualitative changes in the properties of alloys occur if there is transfer of charge between the two bands.

Recently Beeby [11] has developed a theory of alloys in the TBA using total scattering matrices and elements of Green's function techniques. It is interesting to compare his theory with the one developed here by quite different techniques. He predicts a minimum in the middle of the band in the Block limit which we show does not really exist. Beeby does not include the very important charging effects because it is difficult by his method to determine the distribution of the electrons in the alloy. He does, however, find qualitatively much of the behavior of the density of states with alloying found in this paper.

Acknowledgements - The author is very indebted to Professor Mott who suggested the problem and has shown constant interest in it. The many conversations and critical comments by Professor Mott have contributed greatly to the author's understanding of the problem. The author is also indebted to Dr. D. Shoenberg for his hospitality and for arranging the use of facilities during the author's Sabbatical leave.

\section{REFERENCES}

1. N.F. MOTT, Advances in Phys. 13, 325 (1964).

2. P.A. BECK, Electronic Structure and Alloy Chemistry of the Transition Metals (published by Interscience for A.I.M.E. 1964).

3. F. SEITZ, Theory of Metals (published by McGraw-Hill, New York, 1940), pp. 301-303.

4. J.H. WOOD, Phys. Rev. 117, 714 (1960); Quart. Prog. Rep. No. 39, Solid State and Molecular Theory Group, M.I.T., Jan. 15th, p. 79 (1961).

5. J. HUbbard, Proc. Roy. Soc. A276, 238 (1963); Proc. Roy. Soc. A277, 237 (1964); Proc. Roy. Soc. A281, 392 (1964).

6. F.E. HOARE, article in reference 2 .

7. P.A. MOLFF, Phys. Rev. 124, 1030 (1961).

8. P.M. ANDERSON, Phys. Rev. 124, 41 (1961).

9. J.H. VAN VLECK, Rev. Mod. Phys. 25, 220 (1953).

10. J. KANAMORI, Prog. Theor. Phys, 30, 275 (1963).

11. J.L. BEEBY, Phys. Rev. 135, A130 (1964. 